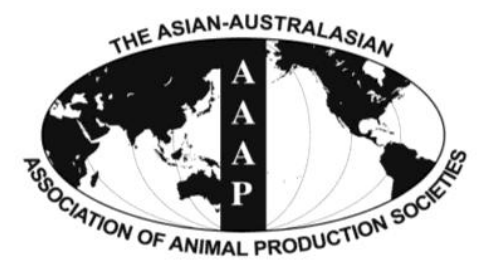

Asian-Aust. J. Anim. Sci.

Vol. 25, No. 3 : 299 - 303

March 2012

www.ajas.info

http://dx.doi.org/10.5713/ajas.2011.11315

\title{
Evaluation of Optimum Genetic Contribution Theory to Control Inbreeding While Maximizing Genetic Response
}

\author{
S.-H. Oh* \\ North Carolina Agricultural and Technical State University, Greensboro, NC, USA
}

\begin{abstract}
Inbreeding is the mating of relatives that produce progeny having more homozygous alleles than non-inbred animals. Inbreeding increases numbers of recessive alleles, which is often associated with decreased performance known as inbreeding depression. The magnitude of inbreeding depression depends on the level of inbreeding in the animal. Level of inbreeding is expressed by the inbreeding coefficient. One breeding goal in livestock is uniform productivity while maintaining acceptable inbreeding levels, especially keeping inbreeding less than $20 \%$. However, in closed herds without the introduction of new genetic sources high levels of inbreeding over time are unavoidable. One method that increases selection response and minimizes inbreeding is selection of individuals by weighting estimated breeding values with average relationships among individuals. Optimum genetic contribution theory (OGC) uses relationships among individuals as weighting factors. The algorithm is as follows: i) Identify the individual having the best EBV; ii) Calculate average relationships $\left(\bar{r}_{j}\right)$ between selected and candidates; iii) Select the individual having the best EBV adjusted for average relationships using the weighting factor $k, E B V^{*}=E B V_{j}\left(1-k \overline{r_{j}}\right)$. iv) Repeat process until the number of individuals selected equals number required. The objective of this study was to compare simulated results based on OGC selection under different conditions over 30 generations. Individuals $(n=110)$ were generated for the base population with pseudo random numbers of $\mathrm{N} \sim(0,3)$, ten were assumed male, and the remainder female. Each male was mated to ten females, and every female was assumed to have 5 progeny resulting in 500 individuals in the following generation. Results showed the OGC algorithm effectively controlled inbreeding and maintained consistent increases in selection response. Difference in breeding values between selection with OGC algorithm and by EBV only was $8 \%$, however, rate of inbreeding was controlled by $47 \%$ after 20 generation. These results indicate that the OGC algorithm can be used effectively in long-term selection programs. (Key Words : Genetic Contribution Theory, Genetic Response, Breeding Value)
\end{abstract}

\section{INTRODUCTION}

Selection response has been improved continuously through advanced genetic selection programs recently although increase of inbreeding rate has been issued at the same time (Weigel and Lin, 2002). Inbreeding is the matings of individuals with ancestry in common and is based on the average relationship among all individuals in the population. Inbreeding increases homozygosity of alleles. Therefore, individuals with high inbreeding coefficients have a greater probability of homozygous alleles. Inbreeding has been used to develop uniform herds of animals with more homozygous genotypes, but increased inbreeding may cause side effects such as reproductive atrophy resulting in stillbirth or deformity. In pigs,

\footnotetext{
* Corresponding Author : S.-H. Oh. Tel : +1-336-334-7672, Fax : +1-336-334-7288, E-mail : soh@ncat.edu Submitted Sept. 5, 2011; Accepted Dec. 5, 2011; Revised Jan. 9, 2012
}

reduction of litter size due to increased inbreeding can be the result of either decreased conception rate as inbreeding level of sows increase, or loss of vitality of piglets as their inbreeding level increases.

Falconer (1960) has reported on experiments from longterm, full-sib, mating of mice that approximately $40 \%$ of the reduction in litter size due to inbreeding was attributable to reduced fertility of the females and the remaining $60 \%$ to reduced survivability of the young. In cattle, it has been reported that a $1 \%$ increase in the inbreeding coefficient resulted in decrease of milk production by $37 \mathrm{~kg}$ and the production period by 13.1 days in Holstein cows (Smith et al., 1998). In pigs a $10 \%$ increase in inbreeding of young pigs has been reported to reduce litter size by 0.29 while a $10 \%$ increase in inbreeding of the female results in the reduction of 0.40 head per litter (Johnson, 1990). In poultry, Sewalem et al. (1999) have reported that inbreeding depression was not so much for egg production traits, but an 
increase of inbreeding was related to a delay in sexual maturity. It has been reported that $0.7 \%$ of inbreeding coefficients on average increased every year after 23 generations without full- and half-sib matings, which resulted in 3 eggs decrease per year (Ameli et al., 1991).

With this reason, many methods to maximize genetic progress minimizing the rate of inbreeding have been suggested and studied. Weigel (2001) has summarized the methods, which are restrictions on family size, creation of sublines, mating programs, modifications to BLUP, and optimum contribution theory. Optimal inbreeding theories suggest that breeding values would be maximized while minimizing inbreeding. It is said that methods based on optimum contribution theory are more promising among them (König et al., 2010). Although numerous cases were studied, there are few researches suggesting which constraints would be used properly in overall because they rely on specific breeding programs and the individual numbers of foundation population. One of the theories has been suggested by Quinton and Smith (1995), which is based on the consideration of relationship coefficients among selection candidates resulting in their re-ranking. It also depends on the size of the foundation herds. Their method is relatively intuitive than other methods in terms of the factors to be considered in the model such as the number of foundation group, heritability, and weighting factors. However, they just showed the results of 10 generations, which may not be enough to explain the effect for building an inbred line. Also, it was not clear about the level of weighting factors in each gender group with different mating conditions.

The purpose of this study is not to improve the existing method, nor to suggest a new algorithm. It is to evaluate the effect of OGC theory on the inbreeding through the computer simulation of the method suggested by Quinton and Smith (1995), and to find out how much it would control the rate of inbreeding in a closed herd starting 10 sires and 100 dams with different combinations of weighting factors to breeding groups over 20 generations. Livestock producers will have some idea regarding to the optimal combinations of weighting factors in a particular mating condition from the results in this study.

\section{MATERIALS AND METHODS}

Increasing selection response with minimal inbreeding is based on controlling the ranking of individuals weighting selection based on EBVs with average relationships among individuals. Optimum genetic contribution theory (OGC) uses those relationships as weights. Selection based only on EBV from BLUP evaluations results in increased rates of inbreeding especially in long-term selection because the probability is greater that relatives with superior EBVs will be selected together. Optimum genetic contribution as suggested by Quinton and Smith (1995) controlled the increase of inbreeding in a long-term closed miniature pig selection program by considering EBV and the rate of inbreeding between candidates and other individuals in the population. The steps involved in the algorithm are as follows:

i) Identify the individual having the best EBV;

ii) Calculate average relationships $\left(\overline{r_{j}}\right)$ between selected individual(s) and candidate individuals;

iii) Select the individual having the best OGC score with $E B V^{*}=E B V_{j}\left(1-k \overline{r_{j}}\right)$ using the weighting factor $k$;

iv) Repeat the steps until the number of individuals required are reached;

v) Repeat process for the opposite sex.

Quinton and Smith (1995) demonstrated that OGC could maintain genetic improvement comparable to selection on only EBVs in a simulation of 10 generations with $k$ values from 0.5 to 2.0 . In this study, 20 generations were simulated for 25 combinations of $k$ for both males and females where $k$ ranged from 0 to 2.0 and was increased by 0.5 . The simulated trait was litter size with a heritability of 0.1 . One hundred and ten pigs were generated for the base population with pseudo random numbers $\mathrm{N} \sim(0,3)$. Ten pigs were assumed as male, and the rest as female because most of populations show polygamic structures with high mating ratios (Sánchez et al., 2003). Each male was mated to ten females, and every female was assumed to have 5 progeny resulting in 500 individuals for the following generations. Sex ratio was assumed to $1: 1$. Ten males and one hundred females were selected and mated using the OGC algorithm for the next generations. This same process of selection was repeated for 20 generations. This simulation was analyzed without considering the aspect of overlapping generations. Average EBVs and the rates of inbreeding after ten simulations were calculated each generation.

\section{RESULTS AND DISCUSSION}

These results show that the OGC algorithm simulated in this study can control inbreeding effectively while maintaining consistent selection response. Differences in breeding values between different matings with the OGC algorithm and with only EBVs were less than $8 \%$ (Tables 3 and 4 ). However, the rate of inbreeding could be controlled as much as about $47 \%$ in this OGC simulation over 20 generations (Tables 1 and 2).

Table 3 and 4 are average breeding values and standard deviations over 20 generations of selection with different $k$ values. The expected breeding values after 20 generations were about $8 \%$ higher with $k=0$ than $k=2$ for both sexes 
Table 1. The percentage of increase in the rate of inbreeding with different $k$ values for female when $k$ for male $=0$

\begin{tabular}{lrrrrr}
\hline \multirow{2}{*}{ Generation } & \multicolumn{5}{c}{$k$ for female } \\
\cline { 2 - 6 } & 0.0 & 0.5 & 1.0 & 1.5 & 2.0 \\
\hline 1 & 0.0 & 0.0 & 0.0 & 0.0 & 0.0 \\
2 & 2.9 & 2.6 & 2.3 & 1.9 & 1.8 \\
5 & 22.7 & 19.9 & 17.8 & 16.7 & 16.3 \\
10 & 37.5 & 26.5 & 21.4 & 20.6 & 17.5 \\
15 & 49.3 & 28.2 & 24.8 & 21.8 & 19.6 \\
20 & 57.8 & 31.9 & 26.3 & 23.4 & 20.2 \\
\hline
\end{tabular}

Table 2. The percentage of increase in the rate of inbreeding with different $k$ values for male when $k$ for female $=0$

\begin{tabular}{lrrrrr}
\hline \multirow{2}{*}{ Generation } & \multicolumn{5}{c}{$k$ for male } \\
\cline { 2 - 6 } & 0.0 & 0.5 & 1.0 & 1.5 & 2.0 \\
\hline 1 & 0.0 & 0.0 & 0.0 & 0.0 & 0.0 \\
2 & 3.1 & 2.9 & 2.7 & 2.2 & 1.9 \\
5 & 22.7 & 12.1 & 9.9 & 9.4 & 7.6 \\
10 & 37.5 & 13.2 & 10.5 & 9.7 & 8.0 \\
15 & 49.3 & 13.7 & 10.9 & 10.5 & 10.3 \\
20 & 57.8 & 14.9 & 12.5 & 11.7 & 10.6 \\
\hline
\end{tabular}

Table 3. Percent of increases in the rates of inbreeding compared to the case of $k=0.0$ for male and female

\begin{tabular}{lrrrrr}
\hline$k$ & & \multicolumn{4}{c}{$k$ (female) } \\
\cline { 3 - 6 } (male) & Generation & \multicolumn{1}{c}{0.5} & 1.0 & 1.5 & 2.0 \\
\hline 0.5 & 5 & 9.09 & 7.82 & 7.10 & 5.78 \\
& 10 & 10.38 & 10.24 & 9.77 & 9.34 \\
& 15 & 11.12 & 10.83 & 9.99 & 9.87 \\
& 20 & 13.30 & 11.46 & 11.41 & 11.19 \\
1.0 & 5 & 8.80 & 6.95 & 6.93 & 5.40 \\
& 10 & 9.38 & 8.18 & 7.69 & 7.26 \\
& 15 & 9.57 & 8.80 & 8.33 & 7.62 \\
& 20 & 11.02 & 9.42 & 8.85 & 8.81 \\
1.5 & 5 & 6.26 & 5.26 & 5.34 & 4.34 \\
& 10 & 7.22 & 6.61 & 6.21 & 5.49 \\
& 15 & 7.62 & 7.39 & 7.36 & 5.95 \\
& 20 & 9.42 & 8.31 & 7.88 & 7.45 \\
2.0 & 5 & 4.89 & 4.25 & 4.12 & 3.13 \\
& 10 & 5.95 & 5.49 & 5.28 & 4.37 \\
& 15 & 7.10 & 6.27 & 5.60 & 5.31 \\
& 20 & 8.00 & 7.12 & 6.46 & 5.98 \\
\hline
\end{tabular}

Table 4. Changes of average breeding values and standard deviations in male with different $k$ values for female when $k$ for male $=0$

\begin{tabular}{|c|c|c|c|c|c|}
\hline \multirow{2}{*}{ Generation } & \multicolumn{5}{|c|}{$k$ for female } \\
\hline & 0.0 & 0.5 & 1.0 & 1.5 & 2.0 \\
\hline 1 & $3.00 \pm 0.04$ & $3.01 \pm 0.14$ & $2.85 \pm 0.03$ & $2.99 \pm 0.17$ & $2.99 \pm 0.03$ \\
\hline 2 & $3.65 \pm 0.15$ & $3.59 \pm 0.10$ & $3.67 \pm 0.07$ & $3.41 \pm 0.14$ & $3.67 \pm 0.05$ \\
\hline 3 & $4.10 \pm 0.18$ & $4.08 \pm 0.11$ & $4.19 \pm 0.10$ & $3.92 \pm 0.13$ & $4.12 \pm 0.11$ \\
\hline 4 & $4.49 \pm 0.09$ & $4.58 \pm 0.19$ & $4.63 \pm 0.03$ & $4.34 \pm 0.05$ & $4.50 \pm 0.12$ \\
\hline 5 & $4.96 \pm 0.15$ & $4.99 \pm 0.12$ & $5.01 \pm 0.03$ & $4.76 \pm 0.12$ & $4.86 \pm 0.13$ \\
\hline 6 & $5.38 \pm 0.12$ & $5.41 \pm 0.20$ & $5.42 \pm 0.01$ & $5.12 \pm 0.11$ & $5.25 \pm 0.09$ \\
\hline 7 & $5.77 \pm 0.11$ & $5.83 \pm 0.22$ & $5.83 \pm 0.02$ & $5.54 \pm 0.11$ & $5.65 \pm 0.10$ \\
\hline 8 & $6.21 \pm 0.10$ & $6.26 \pm 0.24$ & $6.24 \pm 0.01$ & $5.94 \pm 0.13$ & $6.05 \pm 0.11$ \\
\hline 9 & $6.64 \pm 0.12$ & $6.72 \pm 0.25$ & $6.66 \pm 0.05$ & $6.38 \pm 0.11$ & $6.43 \pm 0.10$ \\
\hline 10 & $7.07 \pm 0.11$ & $7.13 \pm 0.23$ & $7.08 \pm 0.02$ & $6.76 \pm 0.13$ & $6.82 \pm 0.13$ \\
\hline 11 & $7.49 \pm 0.09$ & $7.57 \pm 0.23$ & $7.48 \pm 0.01$ & $7.18 \pm 0.15$ & $7.26 \pm 0.13$ \\
\hline 12 & $7.92 \pm 0.13$ & $7.98 \pm 0.14$ & $7.95 \pm 0.01$ & $7.56 \pm 0.15$ & $7.58 \pm 0.09$ \\
\hline 13 & $8.34 \pm 0.15$ & $8.43 \pm 0.14$ & $8.32 \pm 0.03$ & $7.99 \pm 0.14$ & $7.99 \pm 0.08$ \\
\hline 14 & $8.76 \pm 0.13$ & $8.82 \pm 0.16$ & $8.72 \pm 0.06$ & $8.41 \pm 0.15$ & $8.34 \pm 0.08$ \\
\hline 15 & $9.20 \pm 0.12$ & $9.27 \pm 0.15$ & $9.12 \pm 0.06$ & $8.81 \pm 0.15$ & $8.68 \pm 0.06$ \\
\hline 16 & $9.63 \pm 0.11$ & $9.66 \pm 0.16$ & $9.56 \pm 0.04$ & $9.16 \pm 0.08$ & $8.93 \pm 0.08$ \\
\hline 17 & $10.08 \pm 0.13$ & $10.07 \pm 0.17$ & $9.96 \pm 0.01$ & $9.56 \pm 0.09$ & $9.36 \pm 0.03$ \\
\hline 18 & $10.48 \pm 0.10$ & $10.47 \pm 0.19$ & $10.36 \pm 0.03$ & $9.92 \pm 0.09$ & $9.70 \pm 0.15$ \\
\hline 19 & $10.91 \pm 0.14$ & $10.87 \pm 0.16$ & $10.80 \pm 0.02$ & $10.29 \pm 0.05$ & $10.04 \pm 0.13$ \\
\hline 20 & $11.33 \pm 0.11$ & $11.30 \pm 0.18$ & $11.20 \pm 0.01$ & $10.68 \pm 0.04$ & $10.45 \pm 0.21$ \\
\hline
\end{tabular}


restricting $k=0$ in one sex. However, overall breeding values were increased regardless of $k$ values. Although inbreeding depression was not included in the simulated model, it should not be a problem to see the effect of the OGC algorithm in this study.

Trends of inbreeding coefficients by generation based on male and female (Tables 1 and 2) selections show that the rates of inbreeding were controlled with different $k$ values. As $k$ values are higher, the rates of inbreeding are decreased. When both $k$ values for male and female were zero, the rate of inbreeding of the $20^{\text {th }}$ generation was increased by $57.8 \%$ compared to the base generation; however, when $k$ value was set to 2.0 in one sex, the rates of inbreeding were limited to $20.2 \%$ and $10.6 \%$ increase with $k=0.0$ for male and for female, respectively, in the 20th generation. This is because higher $k$ values emphasize the relationship between candidates, which results in changes of breeding values that would be lower than the original. Therefore, when $k=2.0$ is compared to $k=1.0$, more strict changes in the original ranks of candidates who have greater degrees of relationship occur than in the candidates having less degrees of relationship resulting in a less related group of candidates controlling the rate of inbreeding. In the mean time, the rates of inbreeding were just $9.4 \%$ increase for male selection and $8.3 \%$ increase for female selection with $k=2.0$ in both sexes compared to the case of selection with $k=0.0$ in both sexes (Table 3).
From the results, the impact of $k$ values in male on the level of inbreeding for the next generation was greater than that in the female because 10 of 250 were selected as male and 100 of 250 were selected as female assuming a sex ratio of 1:1 for the 500 simulated progeny. In other words, selection intensity was higher in male than in female population. Therefore, increasing the $k$ value for the males had a larger effect on reducing inbreeding than increasing the $k$ value of females.

Methods of controlling inbreeding and the algorithm procedures have been well summarized by Weigel (2001). The research applying a cost factor to the average relationship in the selected group decreased the rate of inbreeding by 35 to $39 \%$ after 10 generations of selection with just 1 to $3 \%$ reduction of selection response (Wray and Goddard, 1994). This kind of results can be found in several researches. Meuwissen and Goddard (1998) showed that selection with adjusted EBV decreased a genetic response by $4 \%$ with young bulls for AI progeny test programs while reducing a mean relationship among selected individuals by $22 \%$.

The results in this study indicate that the OGC algorithm can be used effectively for the long-term selection program of any closed population. Also, it shows the possibility that inbreeding could be easily controlled by weighting EBV of candidates in each sex group. Software to predict selection responses and rates of inbreeding from

Table 5. Changes of average breeding values and standard deviations in female with different $k$ values for male when $k$ for female $=0$

\begin{tabular}{|c|c|c|c|c|c|}
\hline \multirow{2}{*}{ Generation } & \multicolumn{5}{|c|}{$k$ for male } \\
\hline & 0.0 & 0.5 & 1.0 & 1.5 & 2.0 \\
\hline 1 & $0.51 \pm 0.04$ & $0.59 \pm 0.04$ & $0.53 \pm 0.02$ & $0.50 \pm 0.15$ & $0.56 \pm 0.11$ \\
\hline 2 & $1.84 \pm 0.04$ & $1.93 \pm 0.01$ & $1.79 \pm 0.15$ & $1.77 \pm 0.05$ & $1.81 \pm 0.02$ \\
\hline 3 & $2.59 \pm 0.12$ & $2.61 \pm 0.07$ & $2.55 \pm 0.14$ & $2.51 \pm 0.02$ & $2.48 \pm 0.09$ \\
\hline 4 & $3.16 \pm 0.15$ & $3.20 \pm 0.05$ & $3.00 \pm 0.16$ & $2.93 \pm 0.02$ & $2.94 \pm 0.12$ \\
\hline 5 & $3.62 \pm 0.14$ & $3.69 \pm 0.01$ & $3.46 \pm 0.15$ & $3.37 \pm 0.01$ & $3.35 \pm 0.06$ \\
\hline 6 & $4.12 \pm 0.15$ & $4.09 \pm 0.01$ & $3.96 \pm 0.17$ & $3.77 \pm 0.01$ & $3.73 \pm 0.06$ \\
\hline 7 & $4.57 \pm 0.13$ & $4.46 \pm 0.03$ & $4.34 \pm 0.19$ & $4.16 \pm 0.02$ & $4.14 \pm 0.02$ \\
\hline 8 & $4.99 \pm 0.10$ & $4.83 \pm 0.02$ & $4.75 \pm 0.18$ & $4.58 \pm 0.01$ & $4.54 \pm 0.03$ \\
\hline 9 & $5.38 \pm 0.12$ & $5.26 \pm 0.01$ & $5.15 \pm 0.21$ & $5.01 \pm 0.01$ & $4.94 \pm 0.04$ \\
\hline 10 & $5.83 \pm 0.12$ & $5.63 \pm 0.02$ & $5.54 \pm 0.22$ & $5.42 \pm 0.11$ & $5.42 \pm 0.03$ \\
\hline 11 & $6.27 \pm 0.09$ & $6.05 \pm 0.01$ & $5.92 \pm 0.22$ & $5.78 \pm 0.05$ & $5.76 \pm 0.03$ \\
\hline 12 & $6.70 \pm 0.10$ & $6.43 \pm 0.03$ & $6.35 \pm 0.20$ & $6.19 \pm 0.10$ & $6.13 \pm 0.01$ \\
\hline 13 & $7.11 \pm 0.13$ & $6.81 \pm 0.05$ & $6.77 \pm 0.22$ & $6.59 \pm 0.07$ & $6.53 \pm 0.05$ \\
\hline 14 & $7.55 \pm 0.14$ & $7.21 \pm 0.06$ & $7.10 \pm 0.22$ & $6.95 \pm 0.06$ & $6.98 \pm 0.04$ \\
\hline 15 & $7.96 \pm 0.15$ & $7.64 \pm 0.09$ & $7.49 \pm 0.17$ & $7.34 \pm 0.14$ & $7.33 \pm 0.01$ \\
\hline 16 & $8.37 \pm 0.16$ & $8.03 \pm 0.06$ & $7.90 \pm 0.14$ & $7.73 \pm 0.15$ & $7.73 \pm 0.01$ \\
\hline 17 & $8.80 \pm 0.17$ & $8.41 \pm 0.01$ & $8.34 \pm 0.14$ & $8.12 \pm 0.11$ & $8.14 \pm 0.05$ \\
\hline 18 & $9.25 \pm 0.15$ & $8.78 \pm 0.02$ & $8.72 \pm 0.15$ & $8.50 \pm 0.11$ & $8.57 \pm 0.05$ \\
\hline 19 & $9.66 \pm 0.11$ & $9.18 \pm 0.01$ & $9.14 \pm 0.13$ & $8.88 \pm 0.12$ & $8.96 \pm 0.02$ \\
\hline
\end{tabular}


breeding programs using this theory would be beneficial to the livestock producers as well as animal keepers. Rutten et al. (2002) have developed the software named as "SelAction" to show possible combinations for optimal mating structure. Although inbreeding depression of the trait was not included in the Mendelian random sampling of this simulation study, the results of this simulation would be valuable because the reduction of the rate of inbreeding was 5 times more than the reduction of breeding values. Hinrichs et al. (2006) have developed an algorithm titled in OCSELECT that is more useful when the number of candidates is huge, reducing computing time. The relationship matrix is included in this algorithm as $\mathrm{A}=$ ZApZ $+\mathrm{D}$, where Ap is the relationship matrix of parents, D is a diagonal matrix having variances of Mendelian sampling, $\mathrm{Z}$ is genetic contributions from parents to progeny.

The stimulation model in this study was not perfect because the inbreeding depression of the trait was not included in the Mendelian random sampling model. Many cases about inbreeding depression have been reported. Irish Holstein-Friesian dairy cows that were $12.5 \%$ inbred showed decreases of $61.8,5.3$, and $1.2 \mathrm{~kg}$ in milk, fat, and protein yields, respectively (Parland et al., 2007). This inbred group also was expected to have a $2 \%$ greater chance of dystocia, and a $1 \%$ greater chance of stillbirth as inbreeding may cause reproductive problems. Gulisija et al. (2007) have reported that the range of the rate of inbreeding was 0.6 to $34 \%$ in their study which was nonparametric analysis of the impact of inbreeding on production with 59,778 Jersey cows. In their results, milk yields were affected by inbreeding depression when the rate of inbreeding was greater than $7 \%$. Therefore, inbreeding depression in milk yields would be prevented with $k>1.5$ in both sexes from the results of this study (Table 3).

It is obvious that OGC theory can reduce effectively the rate of inbreeding over generations with a minimal decrease of selection response. Therefore, the results of this study will help livestock producers focus on a plan to decide the starting numbers of foundation animals to construct a breeding herd. In addition, this kind of simulation studies will provide them their effectiveness and optimal conditions in each theory. Further simulation research would be needed to consider inbreeding depression in Mendelian sampling procedure as well as overlapping generations same as commercial selection programs in practice.

\section{REFERENCES}

Ameli, H., D. K. Flock and P. Glodek. 1991. Cumulative inbreeding in commercial White Leghorn lines under longterm reciprocal recurrent selection. Br. Poult. Sci. 32:439-449.

Falconer, D. S. 1960. Introduction to Quantitative Genetics.

Gulisija, D., D. Gianola and K. A. Weigel. 2007. Nonparametric analysis of the impact of inbreeding on production in Jersey cows. J. Dairy Sci. 90:493-500.

Hinrichs, D., M. Wetten and T. H. E. Meuwissen. 2006. An algorithm to compute optimal genetic contributions in selection programs with large numbers of candidates. J. Anim. Sci. 84:3212-3218.

Johnson, R. K. 1990. Inbreeding effects on reproduction, growth and carcass traits. In: Genetics of Swine (Ed. L. R. Young).

König, S., F. Tsehay, F. Sitzenstock, U. U. von Borstel, M. Schmutz, R. Preisinger and H. Simianer. 2010. Evaluation of inbreeding in laying hens by applying optimum genetic contribution and gene flow theory. Poult. Sci. 89:658-667.

Meuwissen, T. H. E and A. K. Sonesson. 1998. Maximizing the response of selection with a predefined rate of inbreeding: overlapping generations. J. Anim. Sci. 76:2575-2583.

Parland, S. Mc., J. F. Kearney, M. Rath and D. P. Berry. 2007. Inbreeding effects on milk production, calving performance, fertility, and conformation in irish Holstein-friesians. J. Dairy Sci. 90:4411-4419.

Quinton, M. and C. Smith. 1995. Comparison of evaluationselection systems for maximizing genetic response at the same level of inbreeding. J. Anim. Sci. 73:2208-2212.

Rutten, M. J. M., P. Bijma, J. A. Woolliams and J. A. M. van Arendonk. 2002. SelAction: Software to predict selection response and rate of inbreeding in livestock breeding programs. J. Hered. 93(6):456-458.

Sánchez, L., P. Bijma and J. A. Woolliams. 2003. Minimizing inbreeding by managing genetic contributions across generations. Genetics 164:1589-1595.

Sewalem, A., K. Johannson, M. Wilhelmson and K. Lippers. 1999. Inbreeding and inbreeding depression on reproduction and production traits of White Leghorn lines selected for egg production traits. Br. Poult. Sci. 40:203-208.

Smith, L. A., B. G. Cassell and R. E. Pearson. 1998. The effects of inbreeding on the lifetime performance of dairy cattle. J. Dairy Sci. 81:2729-2737.

Weigel, K. A. 2001. Controlling inbreeding in modern breeding programs. J. Dairy Sci. 84(E. Suppl.):E177-E184.

Weigel, K. A. and S. W. Lin. 2002. Controlling inbreeding by constraining the average relationship between parents of young bulls entering AI progeny test programs. J. Dairy Sci. 85:23762383.

Wray, N. R. and M. E. Goddard. 1994. Increasing long-term response to selection. Genet. Sel. Evol. 26:431-451. 